OPEN

SUBJECT AREAS:

PEPTIDES

BIOPHYSICAL CHEMISTRY

Received

3 July 2014

Accepted

11 September 2014

Published

10 October 2014

Correspondence and requests for materials should be addressed to

K.M. (miyamoto@ himeji-du.ac.jp)

\section{Structural model of ubiquitin transfer onto an artificial RING finger as an E3 ligase}

\author{
Kazuhide Miyamoto
}

Department of Pharmaceutical Health Care, Faculty of Pharmaceutical Sciences, Himeji Dokkyo University, Hyogo, Japan.

The artificial WSTF PHD_EL5 RING finger was designed via “ $\alpha$-helical region substitution”, and its structural model for the attachment of activated ubiquitin has been demonstrated. Chemical modifications of Cys residues, the circular dichroism spectra, and substrate-independent ubiquitination assays illustrated that the WSTF PHD_EL5 RING finger has E3 activity, and it is ubiquitinated via Lys14. Homology modeling calculations revealed that the WSTF PHD_EL5 RING finger possesses a classical RING fold for specific E2E3 binding. The docking poses of the WSTF PHD_EL5 RING finger with the UbcH5b-ubiquitin conjugate provided insight into its functional E2 interaction and development of ubiquitination at the atomic level. The structural model of the artificial WSTF PHD_EL5 RING finger proposed by the present work is useful and may help to extend the strategy of $\alpha$-helical region substitution.

$\mathrm{T}$

he RING finger domain contains some chelating residues, namely Cys and/or His, and it binds to two zinc atoms $\mathrm{s}^{1-3}$. Protein ubiquitination is an enzymatic cascade consisting of ubiquitin-activating (E1), ubiquitinconjugating (E2), and ubiquitin-ligating (E3) enzymes ${ }^{4,5}$. Most RING fingers function as E3s and transfer activated ubiquitin from E2s to the $\varepsilon$-amino groups of the substrate Lys ${ }^{6}$. The enzymatic activities of E2 and E3 are associated with various diseases such as cancer and Parkinson's disease ${ }^{7-9}$. In fact, overexpression of E2s is associated with tumor development in human cancers ${ }^{10}$. The detection of E2 activity in the ubiquitination system can facilitate the monitoring of tumor progression for diagnostic and prognostic purposes. E2s are attractive targets as tumor markers of various cancers. To detect E2 activity, both an E3 and a substrate are indispensable in the ubiquitination system.

Recently, the " $\alpha$-helical region substitution" method was reported for designing artificial RING fingers ${ }^{11,12}$. E3 RING fingers always possess the essential $\alpha$-helical region for E2-E3 binding ${ }^{13}$. If the $\alpha$-helical region of the EL5 RING finger is inserted into the amino acid sequence between the sixth and seventh zinc ligands of the WilliamsBeuren syndrome transcription factor (WSTF) PHD finger, then the artificial WSTF PHD_EL5 RING finger is created on the basis of the EL5 RING finger (Figure 1). The WSTF PHD_EL5 RING finger functions as E3 and cooperates with the same E2 UbcH5 as the EL5 RING finger ${ }^{12}$. In the absence of a substrate, the EL5 RING finger has no E3 activity, however the WSTF PHD_EL5 RING finger is poly-ubiquitinated via Lys63 of ubiquitin, but not Lys6 and Lys $48^{11}$.

The WSTF PHD_EL5 RING finger is useful for detecting the E2 activity of UbcH5 because its substrate is inessential for ubiquitin transfer. There is growing interest in characterizing the ubiquitination mechanism of artificial RING fingers. However, detailed structural information about artificial RING fingers and their ubiquitination sites at the atomic level is not available. This knowledge is crucial for the molecular design of various artificial RING fingers as E3.

In this study, we investigated the structural features of the artificial WSTF PHD_EL5 RING finger using homology modeling and circular dichroism (CD) spectroscopy. In addition, the ubiquitination site of the WSTF PHD_EL5 RING finger was assessed via mutational analysis of five Lys residues. The present report provides important information about the ubiquitination mechanism of this artificial RING finger.

\section{Results}

Secondary structure of the artificial WSTF PHD_EL5 RING finger. To obtain information on the secondary structure of the WSTF PHD_EL5 RING finger and its five mutants (K4R, K8R, K9R, K14R, and K23R) shown in Figure 1, their CD spectra were recorded at room temperature in Solution A consisting of $20 \mathrm{mM}$ Tris- $\mathrm{HCl}(\mathrm{pH}$ 6.9), $50 \mathrm{mM} \mathrm{NaCl}, 1 \mathrm{mM}$ dithiothreitol, and $50 \mu \mathrm{M} \mathrm{ZnCl}_{2}$. As the WSTF PHD_EL5 RING finger has E3 activity, the synthesized five mutations would be expected to alter the function of the protein and decrease its E3 ligase activity. As shown in Figure 2, all of the CD spectra were suggestive of the typical helical conformation with 


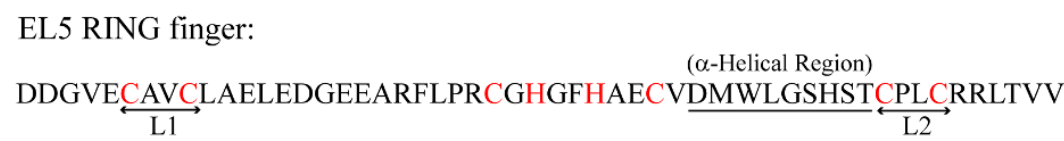

B

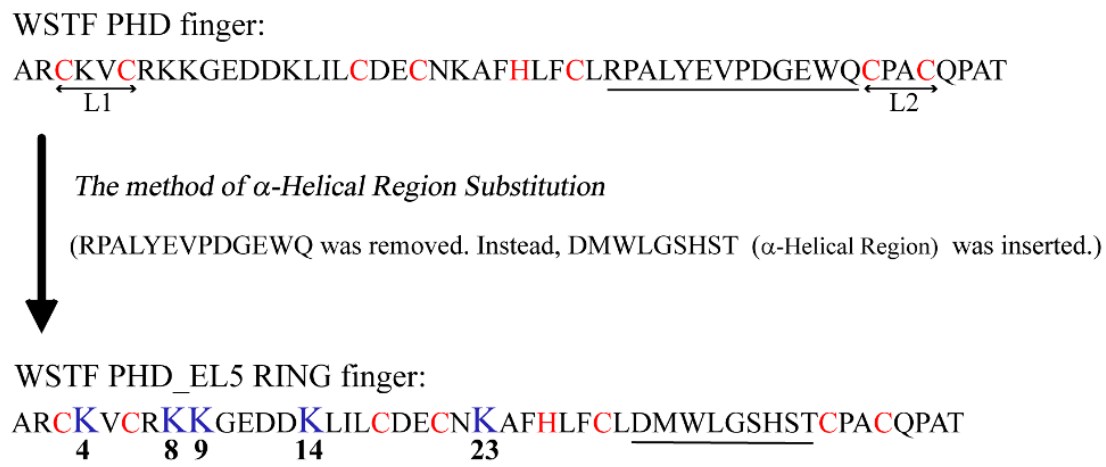

Figure 1 Strategy for the creation of the artificial RING finger as E3 ligase. (A) Amino acid sequence of the EL5 RING finger. (B) Creation of an artificial RING finger by $\alpha$-helical region substitution of the helical region (underlined). Zinc ligands are shown in red. L1 and L2 display the short loop between zinc ligands. Lys residues that were replaced with Arg are shown in blue.

double minima occurring at approximately $205\left(\pi-\pi^{*}\right.$ transitions) and $225 \mathrm{~nm}$ (n- $\pi^{*}$ transitions $)^{14}$. The molar ellipticity occurring at approximately $208 \mathrm{~nm}$ shows the existence of $\alpha$-helical and $\beta$-sheet conformations ${ }^{15}$. However, the $\mathrm{CD}$ spectrum of K23R displayed slight decreases at the negative ellipticities, although the shapes of these spectra were extremely similar. Furthermore, the zinc:protein stoichiometry was estimated by the chemical modification of the Cys residues. The protein-binding zinc ions were released by $p$-hydroxymercuribenzoic acid and quantified using the metallochromic indicator 4-(2-pyridylazo) resorcinol. The concentration of the WSTF PHD_EL5 RING finger were $2.0 \mu \mathrm{M}$, and that of the zinc atoms released by $\mathrm{K} 4 \mathrm{R}, \mathrm{K} 8 \mathrm{R}, \mathrm{K} 9 \mathrm{R}, \mathrm{K} 14 \mathrm{R}$, and $\mathrm{K} 23 \mathrm{R}$ mutants was $4.5,4.4,4.0,3.7$, and $3.5 \mu \mathrm{M}$, respectively. The molar calculated ratios ([Zn]/[protein]) were $2.25,2.20,2.00,1.85$, and 1.75 , respectively. This finding indicates that the artificial RING fingers bind to two zinc atoms for proper folding with $\mathrm{C}_{4} \mathrm{HC}_{3}$-type zinc coordination.
Identification of the ubiquitination site of the artificial RING finger. To identify the ubiquitination site of the WSTF PHD_EL5 RING finger, the E3 activities of the artificial RING finger and its five mutants were assessed via in vitro substrate-independent ubiquitination. The ubiquitin reaction, even in the absence of an E3 ligase, promotes the formation of ubiquitin thioester-linked conjugates of the $\mathrm{E} 2 \mathrm{UbcH} 5 \mathrm{~b}^{16,17}$. The addition of the wild-type artificial RING finger or its mutants led to the accumulation of ubiquitin-E3 conjugates, and mono- and poly-ubiquitination were clearly observed (Figure 3 ). The products corresponding to the higher molecular weight around $148 \mathrm{kDa}$ were observed, and thus the poly-ubiquitination was preferentially on the WSTF PHD_EL5 RING finger. K4R, K8R, and K9R possessed similar E3 activities as the wild-type artificial RING finger. Ubiquitination of the K23R mutant was somewhat weaker than that of the wild-type artificial RING finger. Excluding the K14R mutant, the mutants functioned as

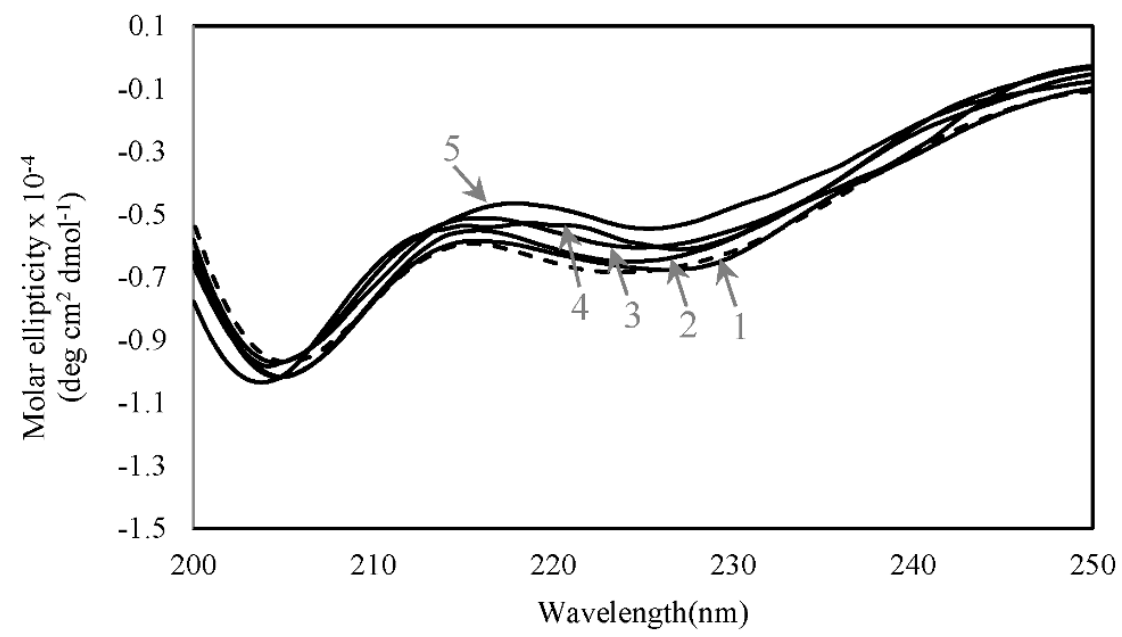

Figure 2 CD spectra of the artificial WSTF PHD_EL5 RING finger and its five mutants. Spectra of $25 \mu \mathrm{M}$ samples were collected in $20 \mathrm{mM}$ Tris-HCl (pH 6.9), $50 \mathrm{mM} \mathrm{NaCl}, 1 \mathrm{mM}$ dithiothreitol, and $50 \mu \mathrm{M} \mathrm{ZnCl}_{2}$ at room temperature. (1) K4R, (2) K8R, (3) K9R, (4) K14R, and (5) K23R are denoted by solid lines, and the dotted line displays the wild-type. 


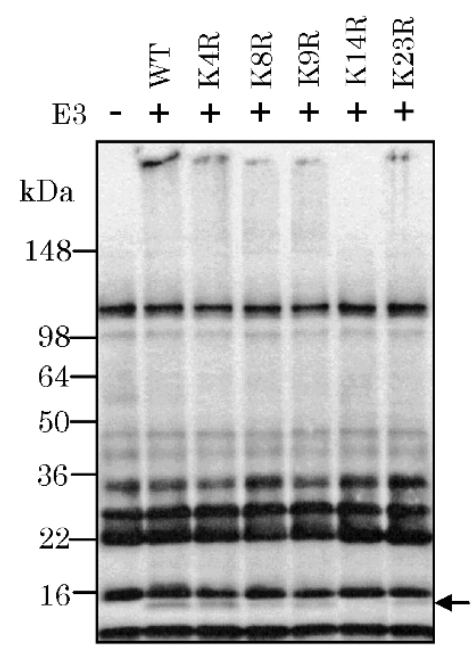

Figure 3 | Ubiquitination of the artificial WSTF PHD_EL5 RING finger and its five mutants. The wild-type (WT) WSTF PHD_EL5 RING finger and its mutants were used in the ubiquitination reaction together with randomly biotinylated ubiquitin, E1, and $\mathrm{E} 2$ ( $\mathrm{UbcH} 5 \mathrm{~b}$ ). The biotinylated ubiquitin on the PVDF membrane was reacted with streptavidin-

horseradish peroxidase solution and a chemiluminescence reagent. The emitted signals were detected using a Luminescent Image Analyzer LAS3000. The arrow shows the product of the mono-ubiquitination, and the molecular weight of the ubiquitinated WSTF PHD_EL5 RING finger is approximately $14569 \mathrm{Da}$.

E3s with cooperating UbcH5b. In addition, mono-ubiquitination of the K14R mutant was not observed in the reaction system. Taken together, these data indicate that Lys14 of the WSTF PHD_EL5 RING finger is essential for mono- and poly-ubiquitination under the present conditions. The spatial location and structural features of Lys14 in the WSTF PHD_EL5 RING finger at the atomic level will be examined later via structural modeling methods.

Structural model of the artificial RING finger. As no structure of the artificial RING finger is available, homology modeling was performed using the program I-TASSER, and the structure of the WSTF PHD_EL5 RING finger was predicted (Supplementary PDB file 1). The built structure has a good C-score of 1.11 and a TM-score of 0.87 for the top model. The C-score indicates high quality of the model predicted by I-TASSER ${ }^{18}$. The appropriate templates (EL5 RING finger (PDB code: 1IYM); FANCL (PDB code: $3 \mathrm{~K} 1 \mathrm{~L}$ ); praja1 RING finger (PDB code: 2LOB)) were automatically collected for the building calculation by I-TASSER. The sequence identities of the whole template chains with the query sequence for EL5 RING finger, FANCL, and praja-1 RING finger are 45, 36, and 28\%, respectively. The resulting three-dimensional structure of the WSTF PHD_EL5 RING finger possessed one $\alpha$-helical region $(\alpha 1)$ and two $\beta$-sheets regions ( $\beta 1$ and $\beta 2$ ), indicative of the typical cross-brace zinc finger as shown in Figure $4^{19-22}$. The structure has a groove for the E2binding site consisting of $\alpha 1, \mathrm{~L} 1$, and L2 regions, which are always present in E3 RING fingers ${ }^{13}$. The quality of the structure was checked using the program PROCHECK ${ }^{23}$. In a Ramachandran plot of the WSTF PHD_EL5 RING finger, non-glycine residues in the most favored regions and the additional allowed regions were located within $85.4 \%$ and $14.6 \%$, respectively. No residue of the structural model presented in this study exists in the disallowed region. The Connolly surface calculated using Discovery Studio 2.1 illustrated that positively charged residues gather at the molecular surface, and thus, the structure of the WSTF PHD_EL5 RING finger exhibits an amphipathic character under the present conditions.

The structure-function relationships of the WSTF PHD_EL5 RING finger provide insight into the structural properties responsible for functional ubiquitination. The structural features of the WSTF PHD_EL5 RING finger were analyzed from the standpoint of its structural similarities with the WSTF PHD and EL5 RING fingers in Figure 5. The WSTF PHD_EL5 RING finger was superimposed over the backbone $\left(\mathrm{N}, \mathrm{C}^{\alpha}, \overline{\mathrm{C}}^{\prime}\right)$ atoms of the WSTF PHD finger with rms deviations of $6.16 \AA$ (Ala1-Thr47) and $2.26 \AA$ (Ala1Leu30), whereas the comparison with the structure of the EL5 RING finger yielded an rms deviation of $2.81 \AA$ (Ala1-Thr47). The structure of the WSTF PHD_EL5 RING finger is similar to that of the WSTF PHD finger in the residues before the helical region. However, the structure of the artificial RING finger as a whole resembles that of the EL5 RING finger in that it has the indispensable helical region for E3 activity.

Possible docking poses. The structural docking of the WSTF PHD_EL5 RING finger and the UbcH5b-ubiquitin conjugate (PDB code: 3A33) was calculated using ZDOCK and Discovery Studio $2.1^{24}$. The possible binding poses were obtained as 1000 structures contacting Lys63 of UbcH5b, which is considered important in $\mathrm{UbcH} 5 \mathrm{~b}-\mathrm{E} 3$ binding $^{25}$. Figure $6 \mathrm{~A}$ presents a best-fit superposition of the ensemble of the three lowest energy structures (Supplementary PDB file 2). The rms deviation is $1.58 \AA$ for the backbone $\left(\mathrm{N}, \mathrm{C}^{\alpha}, \mathrm{C}^{\prime}\right)$ atoms in all residues of the WSTF PHD_EL5 RING finger and the UbcH5b-ubiquitin conjugate. Lys63 of UbcH5b forms a hydrogen bond with Ser36 of the artificial RING finger. Regarding Lys residues for accepting ubiquitin in the WSTF PHD_EL5 RING finger in the lowest energy structure, Lys4 and Lys8
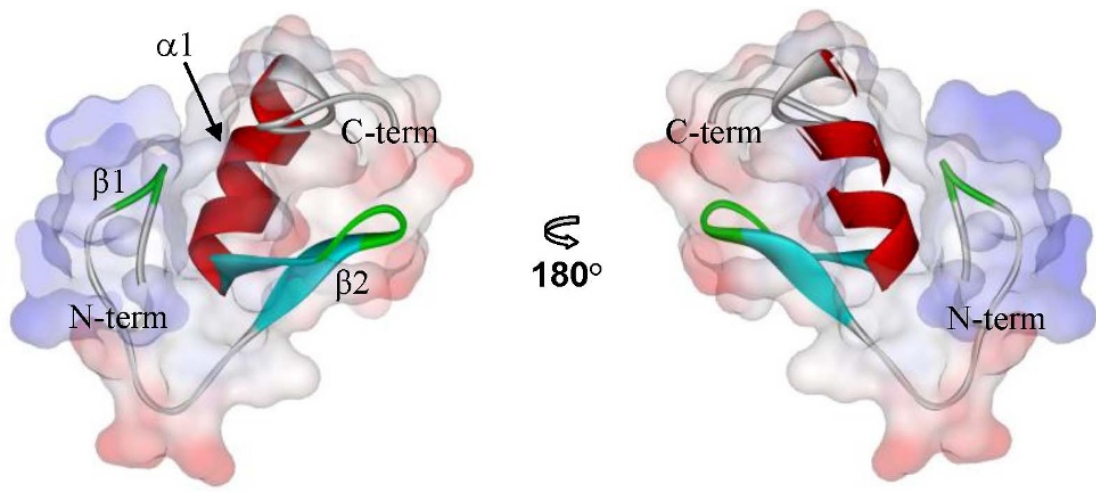

Figure $4 \mid$ Homology modeling structure of the artificial WSTF PHD_EL5 RING finger. The structure was calculated using the program I-TASSER ${ }^{18,34}$. A ribbon diagram was illustrated along a trace of the backbone atoms for the WSTF PHD_EL5 RING finger. Surface representation was calculated using the program Discovery Studio 2.1. The $\alpha$-helical region, $\beta$-sheet regions, and turn regions are shown in red, blue, and green, respectively. Computational results were obtained using software programs from Accelrys Software Inc. 
A

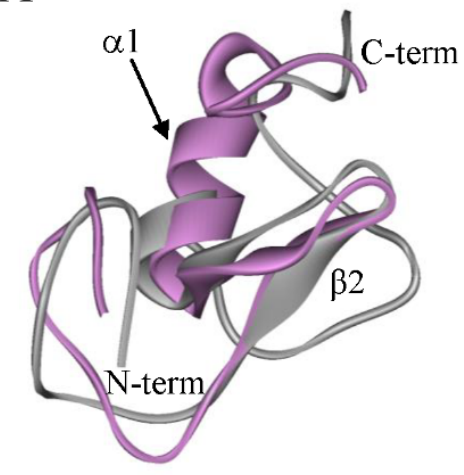

$\mathrm{B}$

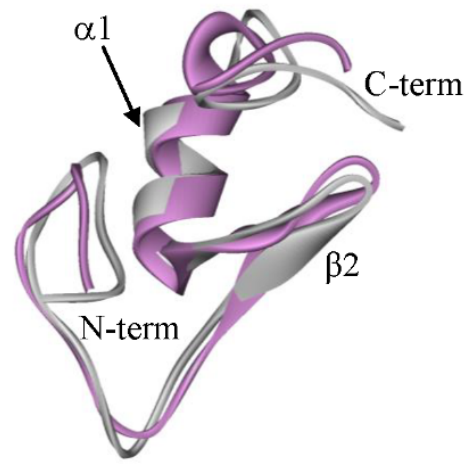

Figure 5 | Structural comparison of the artificial WSTF PHD_EL5 RING finger. The WSTF PHD_EL5 RING finger was superimposed over the backbone atoms for the (A) WSTF PHD and (B) EL5 RING fingers. Ribbon diagrams illustrating a trace of the backbone atoms are used for visualization. The WSTF PHD_EL5 RING finger is shown in magenta. Computational results were obtained using software programs from Accelrys Software Inc.

interact with the residues of UbcH5b (Pro95 and Ala96) and (Arg5 and Lys8), respectively, as shown in Figure 6B. In contrast, Lys9 and Lys 14 in the complex structure possess no contacts with the UbcH5bubiquitin conjugate, and Lys 23 is buried into the hydrophobic core for proper folding. The E2-binding groove of the WSTF PHD_EL5RING finger preferentially contributes to the formation of the E2-E3 complex structure.

\section{Discussion}

The artificial WSTF PHD_EL5 RING finger was created via $\alpha$-helical region substitution on the basis of the EL5 RING finger. Although the substrate for ubiquitin transfer is required in the ubiquitination reaction, the EL5 RING finger, but not the WSTF PHD finger, exhibits E3 activity together with $\mathrm{UbcH} 5 \mathrm{~b}^{12,13,26,27}$. The substituting region derived from the EL5 RING finger confers E3 activity and specific UbcH5b-binding capabilities to the WSTF PHD_EL5 RING finger ${ }^{12}$. In fact, the WSTF PHD_EL5 RING finger was poly-ubiquitinated in cooperation with UbcH5b, as displayed by the substrate-independent ubiquitination assays.

The present study of the WSTF PHD_EL5 RING finger provides an insight into its functional E2 interaction and the development of ubiquitination at the atomic level. Lys residues in the WSTF PHD_EL5 RING finger serve as the scaffolding of the linkages of the poly-ubiquitin chains, even in the absence of the substrate. The homology modeling calculations yielded the three-dimensional structure of the WSTF PHD_EL5 RING finger, in which some Lys residues gather on the molecular surface in an amphipathic manner. The structure of the artificial RING finger contains one helical region $(\alpha 1)$ and two $\beta$-sheets regions ( $\beta 1$ and $\beta 2$ ), which is the typical RING fold for E3 activity ${ }^{22}$. Compared with the WSTF PHD finger, the WSTF PHD_EL5 RING finger has a three-dimensional structure similar to the EL5 RING finger.

The Cys modification experiments revealed that the WSTF PHD_EL5 RING finger and its five mutants (K4R, K8R, K9R, $\mathrm{K} 14 \mathrm{R}$, and K23R) display proper folding with binding to two zinc atoms. The CD spectra revealed that the WSTF PHD_EL5 RING finger and four of its mutants (K4R, K8R, K9R, and K14R) have roughly similar structures. In the K23R mutant, the negative ellipticities diminished slightly at $225 \mathrm{~nm}$ because of replacement of the residue for forming the hydrophobic core in the WSTF PHD_EL5 RING finger. However, mutation of all of the Lys residues did not induce dramatic changes into the unfolding structure.

The structure of the WSTF PHD_EL5 RING finger in complex with the $\mathrm{UbcH} 5 \mathrm{~b}$-ubiquitin conjugate provides crucial knowledge about the functional role and spatial positions of five Lys residues in ubiquitination. The E2-E3 binding interface contains Lys4 and Lys8 of the WSTF PHD_EL5 RING finger. The backbone of Lys9 exists in the proximity of the helical region of $\mathrm{UbcH} 5 \mathrm{~b}$ compared with that of Lys14. Moreover, replacement of Lys14 with Arg clearly abolished both the mono- and poly-ubiquitination reactions, although the structure of the WSTF PHD_EL5 RING finger did not collapse. Taken together, these data illustrate that the WSTF PHD_EL5 RING finger accepts the transferred ubiquitin via Lys14, which promotes the elongation of the poly-ubiquitination chain. In general, the E2-ubiquitin conjugate possesses the remarkable structural flexibility between E2 and ubiquitin, which could lead to facilitate the ubiquitination of the WSTF PHD_EL5 RING finger ${ }^{28}$. In addition, concerning UbcH5b, Arg5, Lys8, Lys63, Pro95, and Ala96 are considered to be involved in the E2-E3 binding interface ${ }^{25}$, and these residues were found in the ZDOCK structure calculated in this study.

In the ubiquitination pathway, tumor progression is associated with E2 activity ${ }^{10}$, and then the application of artificial RING fingers facilitates the detection of E2 activity as a marker of various cancers. Thus, the ubiquitination sites of artificial RING fingers represent an important consideration for the design of E3 ligases, and the present report facilitates the easier creation of these ligases.

In conclusion, the first complex structure calculated for the WSTF PHD_EL5 RING finger displays the mechanism of binding with the $\mathrm{UbcH} 5 \mathrm{~b}$-ubiquitin conjugate and illustrates the indispensability of Lys14 in the ubiquitination reaction. The constructs for avoiding steric hindrance to Lys14 must be preset in various artificial RING fingers. Therefore, the knowledge acquired in this study is useful, and it leads credence to the strategy of $\alpha$-helical region substitution.

\section{Methods}

Peptide synthesis. The artificial WSTF PHD_EL5 RING finger and its five mutants in which Lys residues were replaced with Arg (K4R, K8R, K9R, K14R, and K23R) were synthesized by the standard F-moc solid-phase method (Figure 1). SynProPep products were obtained from Shimadzu Corp. (Kyoto, Japan) for peptide assembly including amide resin. After cleavage with trifluoroacetic acid, peptide purification was performed on a reverse-phase HPLC with Shim-pack C18 column equipment (Shimadzu Corp.). The purity of the peptides was $>98 \%$, and their molecular masses were assessed by MALDI-TOF MS on a Shimadzu AXIMA-TOF ${ }^{2}$. The peptides dissolved in $1 \mathrm{ml}$ of $8 \mathrm{M}$ guanidine- $\mathrm{HCl}$ were dialyzed against degassed Solution A (20 mM Tris- $\mathrm{HCl}$ (pH 6.9), $50 \mathrm{mM} \mathrm{NaCl}, 1 \mathrm{mM}$ dithiothreitol, $50 \mu \mathrm{M} \mathrm{ZnCl}_{2}$ ) overnight at $4{ }^{\circ} \mathrm{C}$ using a Slide-A-Lyzer dialysis cassette (Thermo scientific, Rockford, IL, USA) as described previously ${ }^{12}$.

Stoichiometry of released zinc ions. Concentrations of the WSTF PHD_EL5 RING finger and its mutants were determined spectrophotometrically by the Bradford method against bovine serum albumin. The concentration of the released zinc atoms was determined at room temperature by chemical modification of Cys residues with $p$-hydroxymercuribenzoic acid and 4-(2-pyridylazo)resorcinol ${ }^{29-31}$. The absorbance values (A) were collected at $500 \mathrm{~nm}$, and the concentrations of the zinc ions were estimated using the equation $A=\varepsilon c l$, where the molar absorptivity $(\varepsilon)$ is $6.6 \times$ $10^{4} \mathrm{M}^{-1} \mathrm{~cm}^{-1}$ and the cell length (l) is $1.0 \mathrm{~cm}$. The zinc:protein stoichiometry was calculated on the basis of the amount of the released zinc ions and proteins, as described previously ${ }^{12}$. 
A

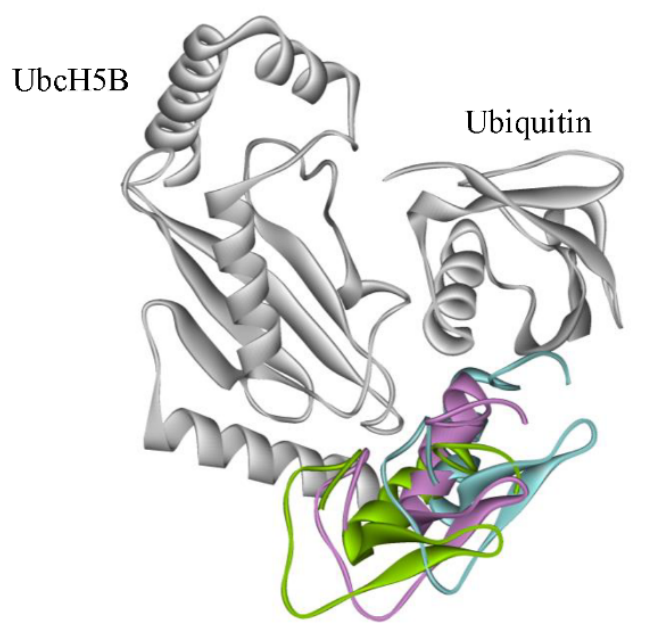

WSTF PHD_EL5 RING finger

B

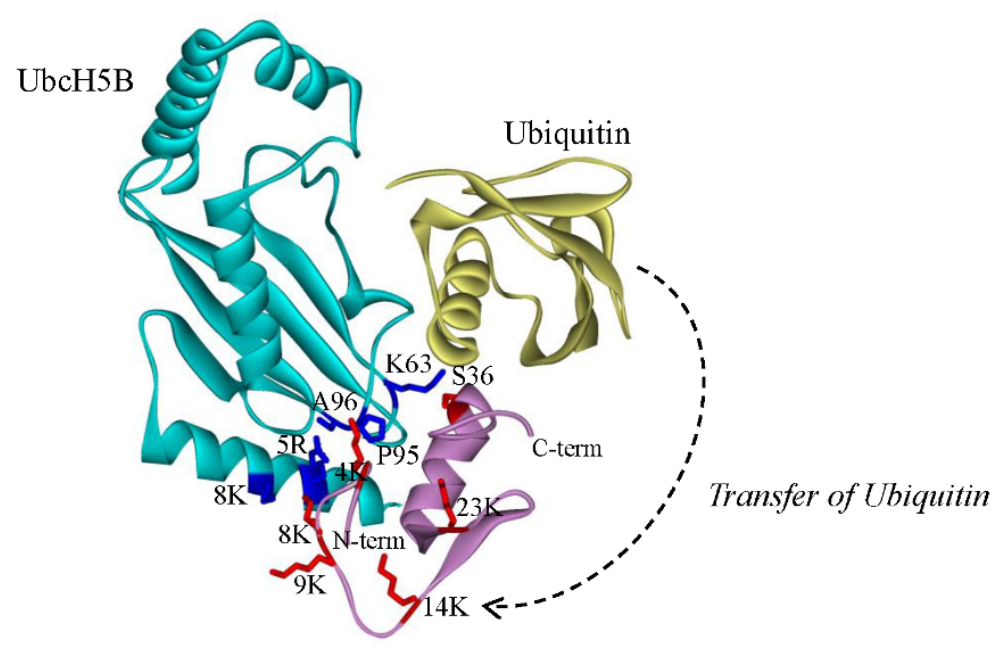

WSTF PHD_EL5 RING finger

Figure 6 Structure of the artificial WSTF PHD_EL5 RING finger in complex with the UbcH5b-ubiquitin congujate. Docking poses were calculated using the program $\mathrm{ZDOCK}^{36}$, and then energy minimization calculations were performed using Discovery Studio 2.1. (A) Ribbon diagrams of the three lowest energy structures of the WSTF PHD_EL5 RING finger. (B) Structural model for transferring ubiquitin in the lowest energy structure. The locations for the five Lys residues of the WSTF PHD_EL5 RING finger and their interacting residues of UbcH5b are shown on the complex structure as well as the interaction of Ser36 and Lys63. The residues on the molecule of the WSTF PHD_EL5 RING finger and UbcH5b are drawn in red and blue, respectively. Computational results were obtained using software programs from Accelrys Software Inc.

CD spectroscopy. The CD experiments were conducted on a JASCO J-805 spectropolarimeter after calibration using d-camphor-10-sulfonate. A 1-mm path length quartz cell was used for $25 \mu \mathrm{M}$ samples. Spectra were obtained at room temperature from 200 to $250 \mathrm{~nm}$ under the conditions of a bandwidth of $1 \mathrm{~nm}$ and a data pitch of $1 \mathrm{~nm}$ at a scan speed of $50 \mathrm{~nm} / \mathrm{min}^{32,33}$. Each CD spectrum was the average of four scans. After subtraction of the solvent spectrum, the CD data were collected by transforming the $\mathrm{CD}$ signal into mean residue molar ellipticity.

In vitro substrate-independent ubiquitination. The WSTF PHD_EL5 RING finger (molecular weight, $5287 \mathrm{Da}$ ) and its five mutants were used as E3 enzymes in substrate-independent ubiquitination reactions. The ubiquitination assays were performed in $50-\mu \mathrm{l}$ reaction volumes of ubiquitination buffer $(20 \mathrm{mM}$ Tris- $\mathrm{HCl}$ ( $\mathrm{pH}$ 6.9), $5 \mathrm{mM} \mathrm{Mg-ATP,} 1 \mathrm{mM}$ dithiothreitol, $20 \mathrm{U} / \mathrm{ml}$ inorganic pyrophosphatase (Sigma, St. Louis, MO, USA), $50 \mu \mathrm{M} \mathrm{ZnCl}_{2}$ ) including $2.5 \mu \mathrm{M}$ biotinylated ubiquitin, $0.1 \mu \mathrm{M}$ human recombinant E1 (His-tagged), and $2.5 \mu \mathrm{g} \mathrm{UbcH5b}$ as E2 (His-tagged). Biotinylated ubiquitin (molecular weight, approximately $9300 \mathrm{Da}$ ), human E1, and UbcH5b were purchased from Enzo Life Sciences (Farmingdale, NY, USA). The mixtures also contained the WSTF PHD_EL5 RING finger or its five mutants at a concentration of $20 \mu \mathrm{M}$. The reaction solutions were incubated at $37^{\circ} \mathrm{C}$ for $60 \mathrm{~min}$ with gentle agitation, and the reaction was stopped by adding non-reducing sodium dodecyl sulfate (SDS) sample buffer. The obtained samples $(10 \mu \mathrm{l})$ were subjected to SDS-PAGE (10-20\%) and transferred to a polyvinylidene difluoride (PVDF) membrane. To detect biotinylated ubiquitin, the membrane was reacted with streptavidin-horseradish peroxidase solution (Vectastain ABC Elite Kit; Vector Laboratories, Burlingame, CA, USA), and then enhanced chemiluminescence (ECL; GE Healthcare, Buckinghamshire, UK) was used as a western blotting detection reagent according to the manufacturer's protocol. The emitted signals were detected using a Luminescent Image Analyzer (LAS-3000; Fujifilm, Tokyo, Japan).

Protein structure modeling. The structure modeling was performed using the ITASSER server (as "Zhang Server"), which consists of multiple threading alignments and iterative template fragment assembly simulations ${ }^{18,34}$. The I-TASSER program was ranked as the best server in the Critical Assessment of protein Structure Prediction experiments. The server generates the most accurate structural predictions 
via a state-of the-art process. The quality of the predicted structures was evaluated by a confidence score (C-score), and a C-score of $>-1.5$ denotes a correct fold. The template modeling score (TM-score) describes the topological similarity between the predicted model and the native structure. A TM-score of $>0.5$ indicates protein pairs with similar folds as well as a more accurate model prediction ${ }^{18}$. The amino acid sequence of the WSTF PHD_EL5 RING finger (Figure 1) was subjected to the ITASSER server, and the obtained structure was validated using PROCHECK ${ }^{23}$. The program Discovery Studio 2.1, San Diego: Accelrys Software Inc., was used to calculate the Connolly surface and prepare drawings of the structures ${ }^{32}$

ZDOCK calculations. The docking calculation was performed using the program ZDOCK $3 \cdot 0.1^{35,36}$. ZDOCK utilizes a fast Fourier transform algorithm to improve the performance for searching in a translational space, and it is an initial-stage rigid-body molecular docking program. The precision of the docking structure calculated by ZDOCK was proven in the Critical Assessment of Prediction of Interactions Challenge ${ }^{35}$. The available structure from the I-TASSER server was used to calculate the docking poses, and the obtained structure was subjected to energy minimization using the Smart Minimizer algorithm (Max steps 200, RMS gradient 0.01) in Discovery Studio $2.1^{32}$. The resulting top three models were used as appropriate candidates.

1. Borden, K. L. RING domains: master builders of molecular scaffolds? J. Mol. Biol. 295, 1103-1112 (2000).

2. Borden, K. L. \& Freemont, P. S. The RING finger domain: a recent example of a sequence-structure family. Curr. Opin. Struct. Biol. 6, 395-401 (1996).

3. Freemont, P. S. The RING finger. A novel protein sequence motif related to the zinc finger. Ann. N Y Acad. Sci. 684, 174-192 (1993).

4. Hershko, A. \& Ciechanover, A. The ubiquitin system. Annu. Rev. Biochem. 67, 425-479 (1998).

5. Varshavsky, A. The ubiquitin system. Trends Biochem. Sci. 22, 383-387 (1997).

6. Joazeiro, C. A. \& Weissman, A. M. RING finger proteins: mediators of ubiquitin ligase activity. Cell 102, 549-552 (2000).

7. Snoek, B. C., de Wilt, L. H., Jansen, G. \& Peters, G. J. Role of E3 ubiquitin ligases in lung cancer. World J Clin Oncol 4, 58-69 (2013).

8. Deshaies, R. J. \& Joazeiro, C. A. RING domain E3 ubiquitin ligases. Annu. Rev. Biochem. 78, 399-434 (2009).

9. Sun, Y. E3 ubiquitin ligases as cancer targets and biomarkers. Neoplasia $\mathbf{8}$, 645-654 (2006).

10. van Ree, J. H., Jeganathan, K. B., Malureanu, L. \& van Deursen, J. M. Overexpression of the E2 ubiquitin-conjugating enzyme UbcH10 causes chromosome missegregation and tumor formation. J. Cell Biol. 188, 83-100 (2010).

11. Miyamoto, K. Ubiquitination of an artificial RING finger without a substrate and a tag. J Pept Sci 18, 135-139 (2012).

12. Miyamoto, K. \& Togiya, K. The creation of the artificial RING finger from the cross-brace zinc finger by alpha-helical region substitution. Biochem Biophys Res Commun 394, 972-975 (2010).

13. Katoh, S., Tsunoda, Y., Murata, K., Minami, E. \& Katoh, E. Active site residues and amino acid specificity of the ubiquitin carrier protein-binding RING-H2 finger domain. J. Biol. Chem. 280, 41015-41024 (2005).

14. Yang, J. T., Wu, C. S. \& Martinez, H. M. Calculation of protein conformation from circular dichroism. Methods Enzymol. 130, 208-269 (1986).

15. Kentsis, A. et al. The RING domains of the promyelocytic leukemia protein PML and the arenaviral protein $\mathrm{Z}$ repress translation by directly inhibiting translation initiation factor eIF4E. J Mol Biol 312, 609-623 (2001).

16. Van Sant, C., Hagglund, R., Lopez, P. \& Roizman, B. The infected cell protein 0 of herpes simplex virus 1 dynamically interacts with proteasomes, binds and activates the cdc34 E2 ubiquitin-conjugating enzyme, and possesses in vitro E3 ubiquitin ligase activity. Proc. Natl. Acad. Sci. U S A 98, 8815-8820 (2001).

17. Hagglund, R. \& Roizman, B. Characterization of the novel E3 ubiquitin ligase encoded in exon 3 of herpes simplex virus-1-infected cell protein 0. Proc. Natl. Acad. Sci. U S A 99, 7889-7894 (2002).

18. Roy, A., Kucukural, A. \& Zhang, Y. I-TASSER: a unified platform for automated protein structure and function prediction. Nat Protoc 5, 725-738 (2010).

19. Brzovic, P. S., Rajagopal, P., Hoyt, D. W., King, M. C. \& Klevit, R. E. Structure of a BRCA1-BARD1 heterodimeric RING-RING complex. Nat. Struct. Biol. 8, 833-837 (2001).

20. Katoh, S. et al. High precision NMR structure and function of the RING-H2 finger domain of EL5, a rice protein whose expression is increased upon exposure to pathogen-derived oligosaccharides. J. Biol. Chem. 278, 15341-15348 (2003).
21. Kostic, M., Matt, T., Martinez-Yamout, M. A., Dyson, H. J. \& Wright, P. E. Solution structure of the $\mathrm{Hdm} 2 \mathrm{C} 2 \mathrm{H} 2 \mathrm{C} 4 \mathrm{RING}$, a domain critical for ubiquitination of p53. J. Mol. Biol. 363, 433-450 (2006).

22. Mercier, P. et al. Structure, interactions, and dynamics of the RING domain from human TRAF6. Protein Sci. 16, 602-614 (2007)

23. Laskowski, R. A., MacArthur, M. W., Moss, D. S. \& Thornton, J. M. PROCHECK: A program to check the stereochemical quality of protein structures. J. Appl. Cryst. 26, 283-291 (1993)

24. Sakata, E. et al. Crystal structure of UbcH5b ubiquitin intermediate: insight into the formation of the self-assembled E2 $\sim$ Ub conjugates. Structure 18, 138-147 (2010).

25. Dominguez, C. et al. Structural model of the UbcH5B/CNOT4 complex revealed by combining NMR, mutagenesis, and docking approaches. Structure $\mathbf{1 2}$ 633-644 (2004).

26. Scheel, H. \& Hofmann, K. No evidence for PHD fingers as ubiquitin ligases. Trends Cell Biol. 13, 285-287; author reply 287-288 (2003).

27. Aravind, L., Iyer, L. M. \& Koonin, E. V. Scores of RINGS but no PHDs in ubiquitin signaling. Cell Cycle 2, 123-126 (2003).

28. Pruneda, J. N. et al. Structure of an E3:E2 Ub complex reveals an allosteric mechanism shared among RING/U-box ligases. Mol Cell 47, 933-942 (2012)

29. Hunt, J. B., Neece, S. H. \& Ginsburg, A. The use of 4-(2-pyridylazo)resorcinol in studies of zinc release from Escherichia coli aspartate transcarbamoylase. Anal. Biochem. 146, 150-157 (1985).

30. Hunt, J. B., Neece, S. H., Schachman, H. K. \& Ginsburg, A. Mercurial-promoted $\mathrm{Zn} 2+$ release from Escherichia coli aspartate transcarbamoylase. J. Biol. Chem. 259, 14793-14803 (1984).

31. Shang, Z., Liao, Y. D., Wu, F. Y. \& Wu, C. W. Zinc release from Xenopus transcription factor IIIA induced by chemical modifications. Biochemistry 28, 9790-9795 (1989).

32. Miyamoto, K. \& Togiya, K. Solution structure of LC4 transmembrane segment of CCR5. PLoS ONE 6, e20452 (2011)

33. Miyamoto, K., Togiya, K., Kitahara, R., Akasaka, K. \& Kuroda, Y. Solution structure of LC5, the CCR5- derived peptide for HIV-1 inhibition. J. Pept. Sci. 16, 165-170 (2010)

34. Zhang, Y. I-TASSER server for protein 3D structure prediction. BMC Bioinformatics 9, 40 (2008).

35. Wiehe, K. et al. ZDOCK and RDOCK performance in CAPRI rounds 3, 4, and 5 Proteins 60, 207-213 (2005).

36. Pierce, B. G. et al. ZDOCK server: interactive docking prediction of proteinprotein complexes and symmetric multimers. Bioinformatics 30, 1771-1773 (2014)

\section{Acknowledgments}

This work was supported by Takeda Science Foundation and JSPS KAKENHI Grant Number 26430147.

\section{Author contributions}

K.M. designed and performed all the experiments. K.M. wrote the main manuscript text and prepared all the figures. All authors reviewed the manuscript.

\section{Additional information}

Supplementary information accompanies this paper at http://www.nature.com/ scientificreports

Competing financial interests: The authors declare no competing financial interests. How to cite this article: Miyamoto, K. Structural model of ubiquitin transfer onto an artificial RING finger as an E3 ligase. Sci. Rep. 4, 6574; DOI:10.1038/srep06574 (2014)

This work is licensed under a Creative Commons Attribution-NonCommercialNoDerivs 4.0 International License. The images or other third party material in this article are included in the article's Creative Commons license, unless indicated otherwise in the credit line; if the material is not included under the Creative Commons license, users will need to obtain permission from the license holder in order to reproduce the material. To view a copy of this license, visit http:// creativecommons.org/licenses/by-nc-nd/4.0/ 